\title{
Effect of Osaterone Acetate Administration on Prostatic Regression Rate, Peripheral Blood Hormone Levels and Semen Quality in Dogs with Benign Prostatic Hypertrophy
}

\author{
Toshihiko TSUTSUI ${ }^{1)}$, Tatsuya HORI ${ }^{1)}$, Motoko SHIMIZU ${ }^{1)}$, Chisa TATSUZAWA ${ }^{1)}$ and Eiichi KAWAKAMI ${ }^{1)}$ \\ ${ }^{1)}$ Department of Reproduction, Nippon Veterinary and Animal Science University, 1-7-1 Kyonan-cho, Musashino-shi, Tokyo 180-8602, \\ Japan
}

(Received 15 March 2000/Accepted 25 December 2000)

\begin{abstract}
The effects of osaterone acetate (OSA), which is an anti-androgen agent being developed as a therapeutic drug for benign prostatic hypertrophy (BPH) in dogs, on the degree of prostatic regression and semen qualities were investigated. Prostatic regression was compared between dogs with and without orchidectomy. Five male beagles aged 5-9 years were used in the experiment. OSA was orally administered at doses of $0.2 \mathrm{mg} / \mathrm{kg}$ and $0.5 \mathrm{mg} / \mathrm{kg}$ for one week. The prostatic regression rate one week after the end of administration was $62.6 \%$ on average. In the orchidectomized group, the mean regression rate one week after orchidectomy was $60.1 \%$. However, the prostate became enlarged 6 months after administration, compared to the size prior to administration. The above findings suggested that OSA is clinically applicable as a therapeutic drug for BPH in dogs, and inhibits prostatic hypertrophy during the early phase.
\end{abstract}

KEY WORDS: canine BPH, osaterone acetate, prostatic volume.

J. Vet. Med. Sci. 63(4): 453-456, 2001

In dogs, the prostate becomes enlarged with age. Benign prostatic hypertrophy (BPH) is observed in $95 \%$ of dogs, and the prostatic weight/body weight is reported to be $1.5-$ $2.0 \mathrm{~g} / \mathrm{kg}[2]$. However, it has been reported that when the prostate develops to $0.7 \mathrm{~g} / \mathrm{kg}$ or higher, even if clinical symptoms are not observed, histological abnormalities are already present [11]. Krawice and Heflin reported [9] that prostatic diseases are observed in $43.5 \%$ of male dogs brought to veterinary hospitals and $\mathrm{BPH}$ is the most prevalent age-related disease among intact male dogs. In treatment of BPH, because the prostate is an androgen (A)dependent organ, orchidectomy [11] or treatment with antiA agents [1,3] is performed. We previously showed that oral administration of chlormadinone acetate (CMA), which is a commercially available anti-A agent for human use, improved clincial symptoms during the early phase of $\mathrm{BPH}$ $[4,10]$. However, we also reported that the size of the prostate returned to the same size as that prior to administration 12 weeks later [10]. Therefore, to inhibit BPH for a prolonged period, concurrent use of a CMA implant in addition to oral CMA administration may be recommended [6, 12]. It has been shown recently that osaterone acetate (OSA), which is being developed as a therapeutic drug for canine $\mathrm{BPH}$, inhibits BPH better than CMA [3, 15]. To dogs with $\mathrm{BPH}, 0.1,0.2,0.5,1.0 \mathrm{mg} / \mathrm{kg}$ of OSA was orally administered for one week, and the regression rate was observed. It was shown that administration of OSA at $0.2 \mathrm{mg} / \mathrm{kg}$ or higher caused marked regression of BPH during the early stage, however, the size of the prostate was evaluated by the area obtained from lateral images of X-ray radiography, not be volume [15]. Tsumagari et al. [14] treated BPH dogs with OSA and showed that the effect persisted for 12 months or longer, emphasizing its superiority over CMA.
However, they did not clarify the degree of prostatic regression or duration of persistance $[14,15]$.

Therefore, in this study, we clarified the duration of inhibition of prostatic hypertrophy by OSA using dogs that met the conditions of prostatic hypertrophy proposed by Wheaton et al. [15], and investigated the possibility of OSA for clinical application. The prostatic regression by OSA administration was also investigated by comparison with that in orchidectomized dogs. To more accurately evaluate the prostatic volume (PV), we measured the prostate using an X-ray computer tomography (CT) system that we reported previously $[11,14]$. We simultaneously observed the effects on peripheral blood hormones and semen quality.

Animals: The experimental dogs were 5 male beagles aged 5-9 years $(6.8 \pm 0.9$ years, mean $\pm S E)$ bred and maintained at Nippon Veterinary and Animal Science University, weighing $6.9-13.5 \mathrm{~kg}(11.3 \pm 0.8 \mathrm{~kg})$. The PV of these experimental dogs obtained by the CT method was 13.4$25.1 \mathrm{~cm}^{3}\left(17.6 \pm 2.3 \mathrm{~cm}^{3}\right)$, which showed hypertrophy. The semen qualities were normal, and the dogs were able to copulate and were fertile. Two experimental dogs were maintained per cage measuring $150 \times 70 \times 60 \mathrm{~cm}$. The dogs were fed commercial dry food (Hill's Canine Maintenance, U.S.A.) in the morning and given drinking water three times a day in the morning, afternoon, and evening. Their general condition was observed every day at feeding and at the times of giving drinking water.

OSA administration: OSA was orally administered at a dose of $0.2 \mathrm{mg} / \mathrm{kg}$ to $2 \mathrm{dogs}$ and $0.5 \mathrm{mg} / \mathrm{kg}$ to $3 \mathrm{dogs}$ for seven days. OSA used in this experiment was in a tablet form (containing $5.0 \mathrm{mg}$ of OSA), which were provided by Teikoku Zoki Pharmaceutical K.K. (Japan).

Orchidectomy: After the experiment of OSA administra- 
tion, orchidectomy was performed on 4 dogs in which the PV had returned to the same size as that prior to administration. Orchidectomy was performed under general anesthesia. As a pretreatment, $0.1 \mathrm{mg} / \mathrm{kg}$ of atropine sulfate was subcutaneously administered followed by intravenous administration of $16-20 \mathrm{mg} / \mathrm{kg}$ thiopental sodium (Ravonal ${ }^{\circledR}$ ) 20 min later, and anesthesia was maintained with isoflurane.

Measurement of $P V$ : PV was measured prior to initiation of OSA administration and four days, one and two weeks, and one month after the administration, then every one month thereafter until 6-7 months. In the orchidectomized group, the PV was measured by the same schedule until two months after orchidectomy. The CT was performed under the same general anesthesia used for the orchidectomy.

Measurements of peripheral blood LH and testosterone (T) levels: Blood samples for measurements of peripheral blood LH and T levels after OSA administration were collected prior to (three days before and on the administration day) and every week after initiation of administration. Blood was sampled three times per day (10:00, 12:00, 14:00). LH and T levels were measured three times per day, and the mean value was regarded as the value of that day. In blood sampling, heparin sodium, an anticoagulant, was used. Three milliters of blood were collected at each sampling from the cephalic vein, and immediately centrifuged at $600 \mathrm{G}$ for $15 \mathrm{~min}$ using a cold centrifuge (KR-20000T, KUBOTA Co., Japan). The plasma was stored at $-30^{\circ} \mathrm{C}$ until hormone measurement. Plasma $\mathrm{LH}$ and T levels were measured by the RIA method we previously reported [5].

Semen quality: Semen quality was examined on the same days as blood sampling. Semen was collected in three fractions by the previously reported [8] procedure. In the semen quality, semen volume, sperm motility, sperm count, sperm viability, sperm abnormality and semen $\mathrm{pH}$ were examined.

Statistical analysis: The results of this experiment were analyzed by Student's $t$-test. A p value less than 0.05 was regarded as significant.

$P V$ : Regarding the volume prior to administration as $100 \%$, the changes in PV in the OSA treatment groups are shown individually in the $0.2 \mathrm{mg} / \mathrm{kg}$ group, and presented as the means $\pm \mathrm{SE}$ in the $0.5 \mathrm{mg} / \mathrm{kg}$ and orchidectomy groups in Fig. 1.

In the 2 animals in the OSA $0.2 \mathrm{mg} / \mathrm{kg}$ treatment group, the PV regressed to $77.7 \%$ and $63.1 \%$ one week after administration, respectively. The maximal regression rate was observed at two weeks. As in the $0.2 \mathrm{mg} / \mathrm{kg}$ group, the maximal regression rate was attained two weeks after administration in the $0.5 \mathrm{mg} / \mathrm{kg}$ group. After the maximal regression rate was attained, $\mathrm{PV}$ gradually increased in both treatment groups, and the prostate had recovered to almost the original size 6 months after administration.

PV rapidly decreased after orchidectomy, and regressed to $34.8 \%$ on average after two months. PV was clearly smaller in the castrated group than in the $0.5 \mathrm{mg} / \mathrm{kg}$ OSAtreated group, however, no difference was observed in the percent decrease between PV one week after the end of OSA administration and that one week after castration.

Peripheral blood LH and T levels: Because there were no differences in either the peripheral blood LH or T level after OSA administration between the two treatment groups, the levels are presented as the means \pm SE of 3 animals in the $0.5 \mathrm{mg} / \mathrm{kg}$ group in Fig. 2.

In the OSA $0.5 \mathrm{mg} / \mathrm{kg}$ group, the $\mathrm{LH}$ level was above 5 $n \mathrm{~g} / \mathrm{m} l$ on many days $1-2$ months after administration. However, the level was maintained at a level of $5 \mathrm{ng} / \mathrm{ml}$ or lower during the period 3-5 months after the end of administration.

The T level prior to OSA administration was 2.3-3.8 $\mathrm{ng} /$ $\mathrm{m} l(3.1 \pm 0.3 n \mathrm{~g} / \mathrm{m} l)$. The peripheral blood $\mathrm{T}$ level during the three months after OSA administration was maintained at $2 \mathrm{ng} / \mathrm{m} l$ or lower, showing a clearly decreased level com-

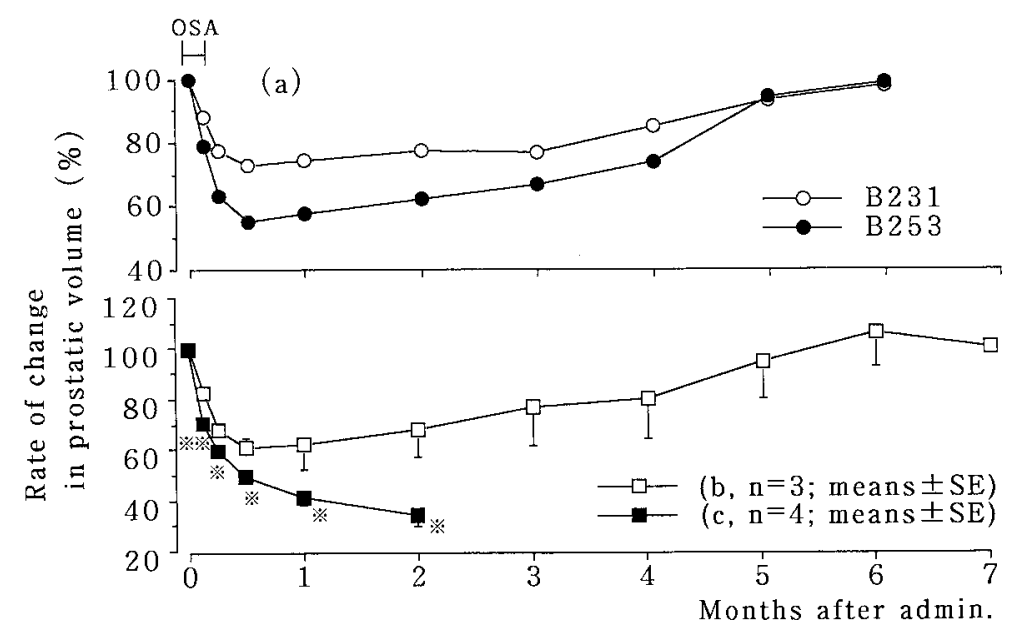

Fig. 1. Changes in prostatic regression rate after one-week administration of OSA at $0.2 \mathrm{mg} / \mathrm{kg}$ (a), $0.5 \mathrm{mg} / \mathrm{kg}$ (b), and after orchidectomy (c). Significantly different from OSA $0.5 \mathrm{mg} / \mathrm{kg}$ administration group $(※ \mathrm{p}<0.05, ※ ※ \mathrm{p}<0.01)$. 


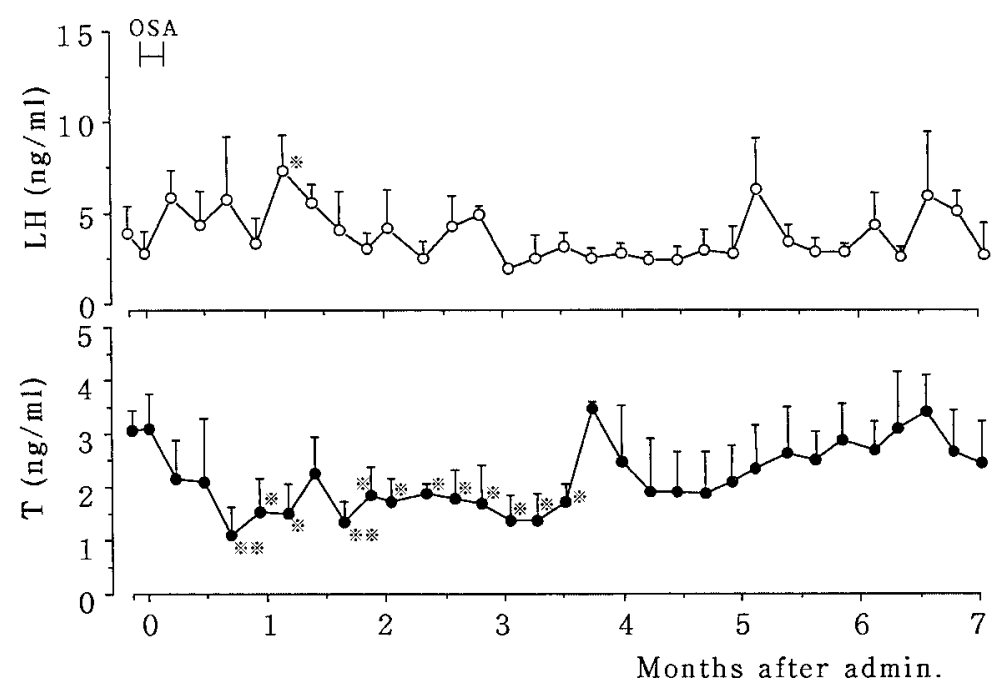

Fig. 2. Changes in plasma LH and T levels (means \pm SE) in dogs with BPH after oral administration with a daily dose of $0.5 \mathrm{mg} / \mathrm{kg}$ OSA for 7 days. Significantly different from pre-treatment $(※ p<0.05, ※ ※ p<0.01)$.

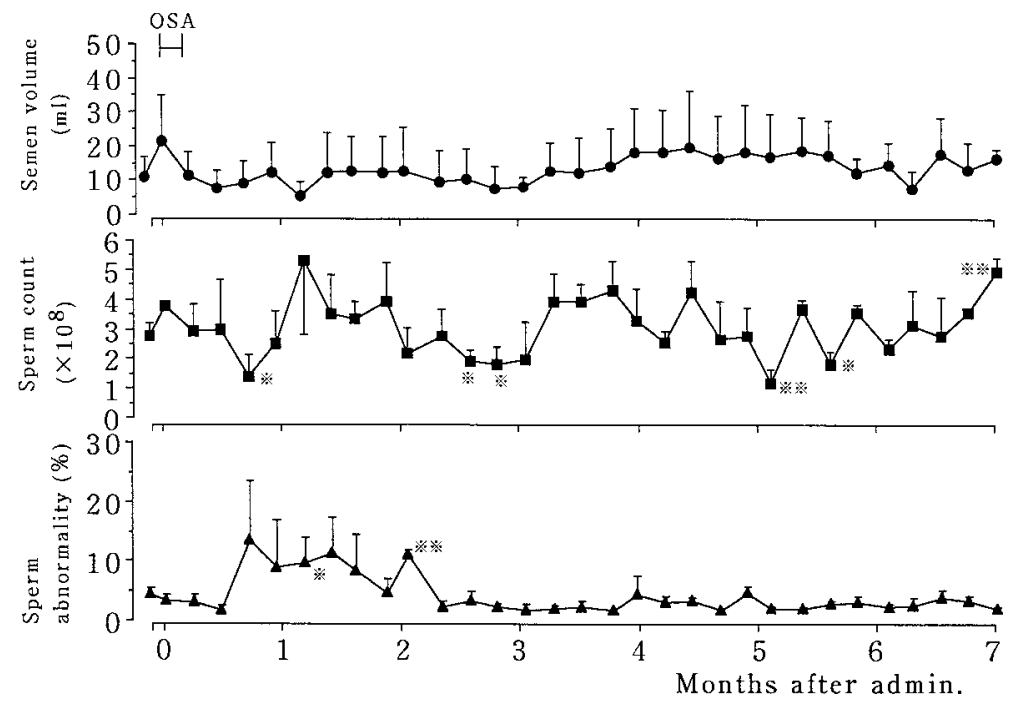

Fig. 3. Changes in semen volume, number of sperm and sperm abnormality (means \pm SE) in dogs with BPH after oral administration with a daily dose of $0.5 \mathrm{mg} / \mathrm{kg}$ (b) OSA for 7 days. Significantly different from pre-treatment $(※ p<0.05, ※ ※$ $\mathrm{p}<0.01)$.

pared to the level prior to administration $(\mathrm{p}<0.05, \mathrm{p}<0.01)$. However, the level slightly increased thereafter, remaining at $2-3 \mathrm{ng} / \mathrm{ml}$.

Semen quality: Because there were no differences in the semen quality after OSA administration between the two treatment groups, the semen quality is presented as the means $\pm \mathrm{SE}$ of three animals in the $0.5 \mathrm{mg} / \mathrm{kg}$ group in Fig. 3.

The semen volume was maintained at about $10 \mathrm{~m} l$ for four months after administration, then increased to $15-20$ $\mathrm{m} l$. The sperm count varied greatly among days of semen collection, but was within a range of $2-4 \times 10^{6}$. The sperm abnormality showed a high level of about $10 \%$ from four weeks after administration for approximately 1.5 months, after which it clearly decreased and was maintained at $5 \%$ or lower. The transiently increased abnormality was observed in the tail region.

No changes were observed in the other semen qualities such as sperm motility and viability and semen $\mathrm{pH}$.

One-week oral administration of OSA at a dose of 0.2 or 
$0.5 \mathrm{mg} / \mathrm{kg}$ to dogs with prostatic hypertrophy showed a marked effect of $62.6 \%$ prostatic mean regression rate one week after the final administration day. In the orchidectomized group, the prostatic mean regression rate one week after orchidectomy was $60.1 \%$, showing that prostatic regression caused by OSA administration occurred soon and rapidly thereafter. Therefore, for the mechanism of PV regression induced by OSA administration during the early phase, Takezawa et al. [13] reported that OSA directly decreased the dihydrotestosterone and androgen receptor contents in the prostate.

The period after OSA administration until the prostate was enlarged to the same size as that prior to administration was six months. These findings suggest that it is desirable to concurrently use a CMA implant with oral OSA administration to inhibit prostatic hypertrophy for a prolonged period in BPH dogs. Tsumagari et al. reported [14] that a one year or longer effect can be expected with oral OSA administration, but they did not describe the size of the prostate. It is necessary to investigate whether the clinical symptoms when the prostatic size returned to the original size are the same as those prior to administration.

For several months after OSA administration, the peripheral blood $\mathrm{T}$ level was maintained at a level clearly lower than that prior to administration. However, the peripheral blood LH level did not change during this period. This finding suggested that due to OSA administration, the testicular secretion of $\mathrm{T}$ directly inhibited the $\mathrm{LH}$ level, not via the pituitary hormone.

Regarding the semen quality, OSA administration transiently increased sperm abnormality. The peripheral blood $\mathrm{T}$ level decreased during this period. This reduction in the $\mathrm{T}$ level changed the secretory discharge in the epididymis, which may have increased the incidence of abnormality in the sperm tail region.

In conclusion, although oral OSA administration transiently decreased the peripheral blood $\mathrm{T}$ level, it did not markedly affect either the LH level or semen quality, suggesting that OSA is clinically applicable as a therapeutic drug for $\mathrm{BPH}$ in dogs.

\section{REFERENCES}

1. Bamberg-Thalem, B. and Linede-Forsberg, C. 1992. J. Am. Vet. Med. Assoc. 39: $264-270$.

2. Berry, S.J., Coffy, D.S. and Ewing, L.L. 1983. Am. J. Physiol. 250: 1039-1046.

3. Habenicht, U.F. and Etreby, M.F. 1987. The Prostate 11: 133143.

4. Kawakami, E., Shimizu, M., Orima, H., Fujita, M., Hori, T. and Tsutsui, T. 1998. Int. J. Androl. 21: 67-73.

5. Kawakami, E., Tsutsui, T. and Ogasa, A. 1990. Jpn. J. Vet. Sci. 52: 179-181.

6. Kawakami, E., Tsutsui, T., Shimizu, M., Orima, H., Fujita, M. and Ogasa, A. 1998. Int. J. Androl. 18: 248-255.

7. Kawakami, E., Tsutsui, T., Shimizu, M., Orima, H., Makanae, Y., Yajima, K. and Ogasa, A. 1998. J. Vet. Med. Sci. 55: 631635.

8. Kawakami, E., Tsutsui, T., Yamada, Y. and Yamauchi, M. 1984. Jpn. J. Vet. Sci. 46: 303-308.

9. Krawiec, D.R. and Heflin, D. 1992. J. Am. Vet. Med. Assoc. 200: 1119-1122.

10. Orima, H., Shimizu, M., Tsutsui, T., Kawakami, E. and Ogasa, A. 1995. J. Vet. Med. Sci. 57: 139-141.

11. Schlottauer, C.F. and Bollman, J.L. 1936. Cornell Vet. 26: 342-349.

12. Shimizu, M., Tsutsui, T., Kawakami, E., Hori, T., Fujita, M., Orima, H. and Ogasa, A. 1995. J. Vet. Med. Sci. 57: 395-399.

13. Takezawa, Y., Ito, K., Suzuki, K., Fukabori, Y., Yamanaka, H., Honma, S., Mieda, M., Hamataki, N. and Kushitani, M. 1995. The Prostate 27: 321-328.

14. Tsumagari, S., Kuyama, M., Kuyama, T., Tanaka, E., Nakagawa, H., Toriumi, H. and Takeishi, M. 1999. J. Jpn. Vet. Med. Assoc. 52: 703-706.

15. Tsutsui, T., Hori, T., Shimizu, M., Orima, H., Kawakami, E. and Fukuda, S. 2000. J. Vet. Med. Sci. 62: 1115-1119.

16. Wheaton, L.G., Dekierk, D.P., Strandberg, J.D. and Coffey, D.S. 1979. Am. J. Vet. Res. 40: 1325-1328. 